\title{
A frequency distributed dissipation model for canopies
}

\author{
N.G. Jacobsen ${ }^{\mathrm{a}, *}$, B.C. McFall ${ }^{\mathrm{b}}$, D.A. van $\operatorname{der} \mathrm{A}^{\mathrm{c}}$ \\ ${ }^{a}$ Harbour, Coastal and Offshore Engineering, Deltares, Delft, The Netherlands \\ ${ }^{b}$ Coastal and Hydraulics Laboratory, US Army Engineer Research and Development Center, Vicksburg, MS, USA \\ ${ }^{c}$ School of Engineering, University of Aberdeen, Aberdeen, UK
}

\begin{abstract}
Existing dissipation models (bulk and frequency distributed) describing the wave attenuation in canopies rely on a characteristic shape of the velocity profile and corresponding characteristic frequency, which is integrated analytically over the height of the canopy. This means that all frequencies higher than the characteristic peak frequency are assigned excessive dissipation, while all frequencies lower than the characteristic peak frequency are assigned insufficient dissipation.

The present work presents a new dissipation model, which is given in a closed form based on the surface elevation spectrum, $S_{\eta}$. The model calculates the frequency dependent dissipation at a given vertical elevation $z$, which is numerically integrated over the height of the canopy. A comparison with existing bulk dissipation models shows that there are large differences between the existing models and the present work. These differences are particularly noticeable for realistic peak enhancements factors for the JONSWAP spectrum (1.0-10.0) and submerged canopies.

A comparison with the frequency distributed dissipation model in the spectral wave model SWAN is also presented and the present model distinguishes itself by naturally incorporating a cut-off frequency above which the dissipation effectively vanishes. This offers a more realistic frequency distribution of the dissipation. The frequency distribution of the dissipation and the existence of a frequency cut-off is verified with experimental data.
\end{abstract}

Keywords: vegetated canopies, spectral energy dissipation

\section{Introduction}

The bottom friction dissipation in spectral wave models (SWAN, MIKE SW, STWAVE, etc.) is commonly frequency dependent, while the dissipation due to vegetation only partially accounts for the frequency of the various wave components. For the bottom friction term (e.g. The SWAN Team, 2013), the shorter waves may locally be in deep water conditions, while the longer waves are in intermediate to shallow water conditions. Consequently, there exists a cut-off frequency above which the dissipation due to bottom friction vanishes. This cut-off frequency is also applicable to dissipation in submerged vegetation canopies, as illustrated in Figure 1. In the following, we will focus on existing dissipation models for waves in canopies and highlight the fundamental assumptions associated with each.

The period-averaged dissipation of wave energy due to rigid stems of vegetation with uniform cross section, $\overline{\mathcal{D}}_{D K H}$, was proposed by Dalrymple et al. (1984) for regular waves:

$$
\overline{\mathcal{D}}_{D K H}=\frac{2}{3 \pi} \rho C_{D} b_{v} N\left(\frac{k g}{2 \omega}\right)^{3} \frac{\sinh ^{3} k h_{v}+3 \sinh k h_{v}}{3 k \cosh ^{3} k h} H^{3}
$$

${ }^{*}$ Corresponding author:

E-mail addresses: Niels.Jacobsen@deltares.nl (N. Jacobsen), Brian.C.McFall@usace.army.mil (B. McFall), d.a.vandera@abdn.ac.uk (D. van der A)
A.

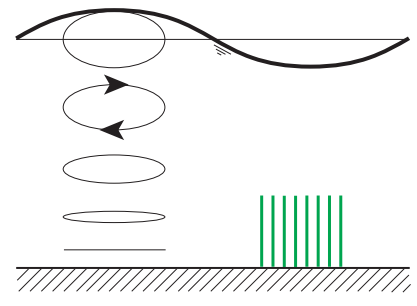

B.

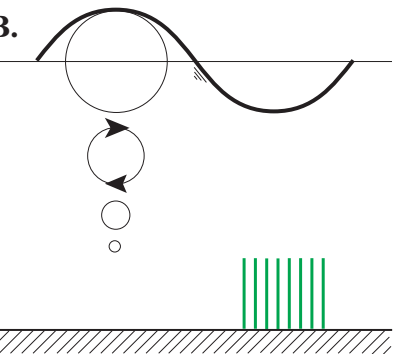

Figure 1: Relationship of particle trajectories from waves in the shallow water (A) and deep water (B) wave regimes with submerged vegetation.

Here, $\rho$ is the density of the fluid, $C_{D}$ is the drag coefficient, $b_{v}$ is the width of the vegetation, $N$ is the number of stems per unit area, $k$ is the linear wave number, $g$ is the acceleration due to gravity, $\omega$ is the cyclic frequency of the wave, $h_{v}$ is the length of the stems, $h$ is the water depth, and $H$ is the local wave height. The dissipation model by Dalrymple et al. (1984) has been used to obtain experimental predictions of $C_{D}$ based on wave attenuation measurements (e.g. Sánchez-González et al., 2011; Anderson and Smith, 2014; Losada et al., 2016), and forms the basis of inclusion of vegetation into wave models based on the mild-slope equations (Cao et al., 2015) and spectral wave models (Suzuki et al., 2012; The SWAN Team, 2013; Anderson and Smith, 2015). Note that Losada et al. (2016) also includes the Doppler-shift due to combined waves and current.

Eq. (1) is based on the assumption that the velocity field 
inside the vegetation canopy may be described by linear wave theory. This assumption has a significant impact on the magnitude of the drag coefficient required to match the dissipation and has been shown in previous studies not to be valid (Lowe et al., 2005; Jacobsen, 2016). This aspect of reduced velocities in the canopy was investigated by Van Rooijen et al. (2016), who showed that a constant drag coefficient across a number of experimental tests could be applied, if the velocity reduction within the canopy is accounted for. Jadhav et al. (2013) used an in-canopy velocity reduction factor similar to Lowe et al. (2005) and derived a frequency dependent drag coefficient for application to the frequency dependent wave energy dissipation model of Chen and Zhao (2012) to numerically model field data collected in a storm. The model of Chen and Zhao (2012) in relation to the present work is discussed in more detail in Section 5.3 .

Mendez and Losada (2004) extended Eq. (1) to account for the dissipation in irregular waves. The dissipation was included in a wave transformation model with no frequency information, thus a bulk dissipation (integrated over all frequencies) was required. They arrived at the following expression:

$\overline{\mathcal{D}}_{M L}=\frac{2}{3 \pi} \rho \tilde{C}_{D} b_{v} N\left(\frac{k_{p} g}{2 \omega_{p}}\right)^{3} \frac{\sinh ^{3} k_{p} h_{v}+3 \sinh k_{p} h_{v}}{3 k_{p} \cosh ^{3} k_{p} h} \cdot \frac{3 \sqrt{\pi}}{4} H_{r m s}^{3}$

Here, $\tilde{C}_{D}$ is a representative drag coefficient for all frequencies, sub-index $p$ refers to quantities based on the peak wave period and $H_{r m s}$ is the root-mean-square wave height. Two assumptions are introduced to arrive at Eq. (2): (i) The shape of the velocity profile is characterised by the peak wave period, $T_{p}$. (ii) The wave heights are Rayleigh distributed and derived from a narrow-banded spectrum. Mendez and Losada (2004) validated their model against an experimental campaign with irregular waves (Lovas, 2000).

Eq. (2) was subsequently adopted in SWAN by Suzuki et al. (2012), where the frequency dependent dissipation was simply related to the distribution of the wave energy density:

$$
\overline{\mathcal{D}}_{S W A N}(f, \theta)=\frac{S_{\eta}(\omega, \theta)}{m_{0}} \overline{\mathcal{D}}_{b u l k}
$$

Here, $S_{\eta}$ is the distribution of the wave energy density over cyclic frequencies $(\omega)$ and direction $(\theta)$ and $m_{0}$ is the total wave energy density. Suzuki et al. (2012) gave $\overline{\mathcal{D}}_{\text {bulk }}$ in a form similar to Eq. (2):

$\overline{\mathcal{D}}_{\text {bulk }}=\frac{1}{2 g \sqrt{\pi}} \rho \tilde{C}_{D} b_{v} N\left(\frac{k_{m 0} g}{2 \omega_{m 0}}\right)^{3} \frac{\sinh ^{3} k_{m 0} h_{v}+3 \sinh k_{m 0} h_{v}}{3 k_{m 0} \cosh ^{3} k_{m 0} h} H_{r m s}^{3}$

Suzuki et al. (2012) replaced $\omega_{p}$ with $\omega_{m 0}$ and $k_{p}$ with $k_{m 0}$ :

$$
\begin{aligned}
k_{m 0} & =\left(\frac{1}{m_{0}} \int_{0}^{2 \pi} \int_{0}^{\infty} \frac{1}{\sqrt{k}} S_{\eta} \mathrm{d} \omega \mathrm{d} \theta\right)^{-2} \\
\omega_{m 0} & =\left(\frac{1}{m_{0}} \int_{0}^{2 \pi} \int_{0}^{\infty} \frac{1}{\omega} S_{\eta} \mathrm{d} \omega \mathrm{d} \theta\right)^{-1} \\
m_{0} & =\int_{0}^{2 \pi} \int_{0}^{\infty} S_{\eta} \mathrm{d} \omega \mathrm{d} \theta
\end{aligned}
$$

Sub-index ' $m 0$ ' refers to spectral mean wave quantities. It should be noted that $k_{m 0}$ and $\omega_{m 0}$ do not necessarily satisfy the linear dispersion relation. The assumptions in Suzuki et al. (2012) are that the dissipation on a given frequency is related to (i) the energy density content on that particular frequency and (ii) the velocity profile is described by the mean wave period, $T_{m 0}=2 \pi / \omega_{m 0}$, and mean wave number, $k_{m 0}$. Irrespective of the difference in characteristic wave number and cyclic frequency, Suzuki et al. (2012) still obtained a good correspondence between their dissipation model and that by Mendez and Losada (2004). The cause of this good correspondence is discussed in Section 3.3.

An alternative approach to classify the energy dissipation in a canopy was taken by Maza et al. (2015), who fitted the wave height decay to

$$
\frac{H}{H_{0}}=\frac{1}{1+\beta x} \quad \text { and } \quad \frac{H_{r m s}}{H_{r m s, 0}}=\frac{1}{1+\beta^{\prime} x}
$$

for regular and irregular waves, respectively. Subscript " 0 " refers to the beginning of the canopy. The $\beta$ value was introduced by Dalrymple et al. (1984) and includes all effects of water depth, vegetation height, canopy density, dimensions of the vegetation and the drag coefficient into one parameter. The parameter $\beta^{\prime}$ is the decay factor similar to $\beta$, however, describing the decay in $H_{r m s}$. Finally, $\mathrm{Hu}$ et al. (2014) evalauted the drag coefficient for a rigid canopy based on force measurements and in-canopy velocity measurements, i.e. incorporating the effect of velocity reduction.

The goal of the present work is to investigate the consequence of the assumptions leading to Eq. (3): (i) Linear wave theory is applicable inside the canopy. (ii) A characteristic velocity profile can be applied to decouple the vertical variation of the velocity profile and the frequency distribution of the wave energy density. (iii) The dissipation at a given frequency is proportional to the energy density at that particular frequency.

The paper is organised as follows: In Section 2 a new frequency dependent dissipation model is presented, which accounts for the frequency effect on the velocity profile and - in part - on the velocity reduction within the canopy. In Section 3 the new model is compared to the dissipation formulations $\overline{\mathcal{D}}_{M L}$ and $\overline{\mathcal{D}}_{S W A N}$ to quantify the effect on the frequency distribution. Finally, the existence of a frequency cut-off is verified against new experimental data in Section 4. Discussions and conclusions are hereafter presented. 


\section{Spectral averaging}

The new dissipation model presented in this section is based on a linear super-position of the velocity contribution from all frequencies. First, the bulk dissipation per vertical layer is formulated and this is subsequently described as a dissipation per vertical layer and per frequency component. Finally the vertical integration of the frequency distributed dissipation is formulated.

\subsection{Resistance and velocity field}

The spectral distribution of the surface elevation, $S_{\eta}$, is the starting point for the dissipation model. The associated velocity spectrum, $S_{u}$, is given as

$$
S_{u}=\left(\frac{\omega \cosh k(z+h)}{\sinh k h}\right)^{2} S_{\eta}
$$

where the multiplier before $S_{\eta}$ follows from linear wave theory. The velocity amplitude associated with the $i$ 'th cyclic frequency reads $u_{m, i}=\sqrt{2 \Delta f S_{u, i}}$ where $\Delta f$ is the frequency discretisation.

The velocity field, $u$, at a given level $z$ is then written as

$$
u(z, t)=\sum_{i} u_{m, i}(z) \cos \left(\omega_{i} t+\phi_{i}\right)
$$

where $\phi_{i}$ is a random phase. Eq. (9) assumes that the velocity field is not influenced by the presence of the canopy.

The drag resistance at a given height $z$ within the canopy is given as:

$$
F_{v}=\frac{1}{2} \rho N b_{v} C_{D} \alpha_{u}^{2} u|u|
$$

where $F_{v}$ is the force per unit horizontal area at level $z$ and $\alpha_{u}$ is a velocity reduction factor (Lowe et al., 2005; Van Rooijen et al., 2016).

The flow resistance due to added mass (inertia) is neglected in Eq. (11), which follows from the observation that the average dissipation due to inertial forces $(\overline{u \partial u / \partial t}=0)$ vanishes following orthogonality for linear velocity fields. The inertia is nonetheless important to account for, when $\alpha_{u}$ is evaluated (Lowe et al., 2005).

For now, it is assumed that $C_{D}$ and $\alpha_{u}$ are frequencyindependent. This is likely a crude approximation, but this is chosen in this work due to the lack of a suitable formulation.

\subsection{Dissipation per layer}

The average wave dissipation per unit time at a given height over the bottom is given as

$$
\bar{d}_{v}(z)=\overline{F_{v} \alpha_{u} u}=\overline{\frac{1}{2} \rho N b_{v} C_{D} \alpha_{u}^{3} u^{2}|u|}=\overline{\Gamma \sum^{2}\left|\sum\right|}
$$

where the short-hand $u=\sum$ is used to stress the complexity of this expression. Here, $\Gamma=1 / 2 \rho N b_{v} C_{D} \alpha_{u}^{3}$ is introduced for convenience.

A single frequency: A single frequency spectrum $(u=$ $u_{1} \sin t$ ) is investigated initially to gain insight. A cyclic frequency of $\omega=1 \mathrm{rad} / \mathrm{s}$ is used for convenience. The Fourier transform of $|u|$ is derived to evaluate $\bar{d}_{v}$ :

$$
|u|=u_{1}\left(\frac{2}{\pi}-\frac{4}{\pi} \sum_{m=1}^{\infty} \frac{\cos 2 m t}{4 m^{2}-1}\right)
$$

For the single frequency the dissipation reads

$$
\bar{d}_{v}(z)=\overline{\Gamma u^{2}|u|}=\overline{\Gamma u_{1} u^{2}\left(\frac{2}{\pi}-\frac{4}{3 \pi} \cos 2 t\right)}
$$

since the remaining terms in the Fourier transform cancel out due to orthogonality. It is observed that the mean of the Fourier transform $(2 / \pi)$ provides a constant scale to $\overline{u^{2}}$ and the Fourier transform produces higher harmonics, which has a finite contribution when averaged with $u^{2}$, since $u^{2}=1 / 2 u_{1}^{2}(1-\cos 2 t)$. Finally, the Fourier transform of $|u|$ contains an infinite number of frequencies with finite energy.

Arbitrary number of frequencies: After investigating a single frequency, the case of an arbitrary number of frequencies is investigated. The bulk dissipation per layer now becomes

$$
\bar{d}_{v}=\Gamma \overline{\sum_{i} \sum_{j} u_{i} u_{j}\left|\sum\right|}
$$

where $u_{i}=u_{m, i} \cos \left(\omega_{i} t+\phi_{i}\right)$. Eq. (15) may be decomposed into contributions for each wave-wave interaction

$$
\bar{d}_{v, i j}=\overline{\Gamma u_{i} u_{j}\left|\sum\right|}
$$

Applying the lesson from the single frequency (Eq. (13)), it is realised that $\left|\sum\right|$ must contain finite energy on all frequencies in the spectrum including $\omega=0$, consequently it is expected that $\bar{d}_{v, i j} \neq 0$ for $i \neq j$, because both $u_{i}$ and $u_{j}$ will observe frequency components in $\left|\sum\right|$ to which they are orthogonal. Hence, the principal of orthogonality cannot be applied, due to the term $\left|\sum\right| .^{1}$

First of all, the diagonal (self-self) contribution to the dissipation is investigated, i.e. $i=j$. Based on numerical experimentation, it was found that

$$
\bar{d}_{v, i i} \simeq \frac{1}{2} \Gamma u_{m, i}^{2} \overline{\left|\sum\right|}=\Gamma \Delta f S_{u, i} \overline{\left|\sum\right|}
$$

\footnotetext{
${ }^{1}$ It is deemed outside the scope of this work to evaluate the exact mathematical expressions for $\Sigma^{2}\left|\sum\right|$ for an arbitrary number of frequencies and random phases, thus the remainder of this work relies on numerical approximations and validation, which are subsequently provided in closed form based on $S_{u}$.
} 
The last equality follows from $u_{m, i}=\sqrt{2 \Delta f S_{u, i}}$. The use of $\simeq$ is applied because the numerical analysis suggests this relationship holds within $\pm 2 \%$ for sufficient long time series. The error of $\pm 2 \%$ is based on single realisations of random phases, while averaging over many realisations results in

$$
\left\langle\bar{d}_{v, i i}\right\rangle_{\phi}=\Gamma \Delta f S_{u, i} \overline{\sum \mid}
$$

Here, $\langle\cdot\rangle_{\phi}$ means algebraic averaging over several realisations of the random phases. Eq. (18) suggests that $\left\langle\bar{d}_{v, i i}\right\rangle_{\phi}$ can be described in a closed form if $\overline{\left|\sum\right|}$ may be evaluated with $S_{u}$ or $S_{\eta}$. The formulation of $\left|\sum\right|$ was pursued with numerical experimentation and it was found that

$$
\overline{\left|\sum\right|} \simeq \sqrt{\frac{2 m_{u, 0}}{\pi}} \text { and }\left\langle\overline{\left.\left|\sum\right|\right\rangle_{\phi}}=\sqrt{\frac{2 m_{u, 0}}{\pi}}\right.
$$

Here, $m_{u, 0}$ is the zeroth moment of the velocity spectrum:

$$
m_{u, 0}=\int_{0}^{\infty} S_{u} \mathrm{~d} f
$$

An example of the dependency of Eq. (19) on the number of wave periods is depicted in Figure 2. The error quantity

$$
\epsilon=\sqrt{\frac{\pi}{2}} \frac{\overline{\left|\sum\right|}}{\sqrt{m_{u, 0}}}-1
$$

is depicted based on evaluations for 9 wave periods, 4 values of the peak enhancement factor (JONSWAP), 5 values of $z / h, 3$ water depths, and 500 realisations of the random phases; in total 270,000 evaluations. It is seen that the standard deviation of $\epsilon$ decreases with increasing number of waves in the time series $\left(N_{w}\right)$. For all cases, the mean of $\epsilon$ is less than $0.3 \%$ from the closed form expression in Eq. (19). For $N_{w}=1,000$ the mean of $\epsilon$ is $0.01 \%$.

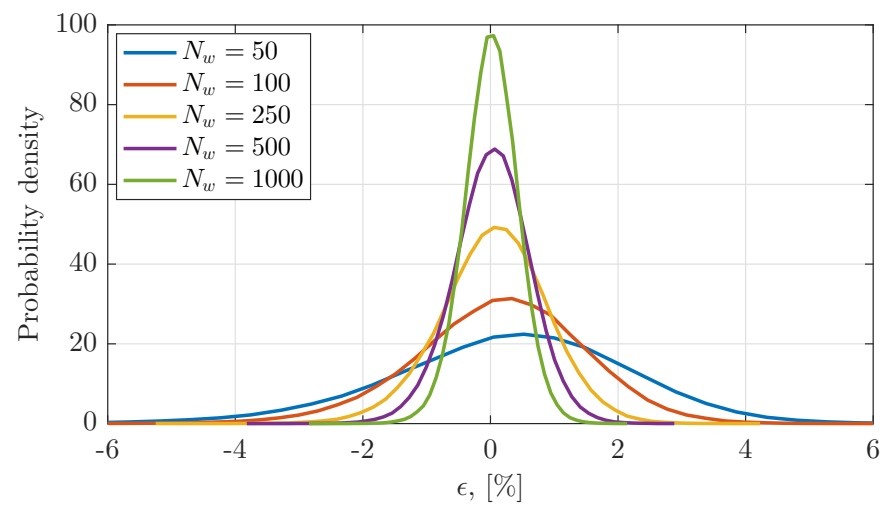

Figure 2: The probability density function of $\epsilon$ (Eq. (21)) as a function of the number of waves $\left(N_{w}\right)$ in each realisation of $u$.

Consequently, the contribution to the bulk dissipation from the self-self terms at a given vertical level $z$ reads

$$
\sum_{i} \bar{d}_{v, i i}=\sum_{i} \Gamma \Delta f S_{u, i} \sqrt{\frac{2 m_{u, 0}}{\pi}}
$$

which is in a closed form based on the velocity spectrum (and the surface elevation spectrum $S_{\eta}$ through Eq. (9)).

The contribution from the off-diagonal terms (cross-terms) must also be formulated to obtain the total dissipation. In spectral wave models, like SWAN, the dissipation is lumped into each frequency and the distribution of the cross-terms are thus added to the diagonal dissipation terms. Consequently, the cross-terms are described for the $i$ 'th frequency as

$$
\bar{d}_{v, i i}^{\times}=\sum_{j, j \neq i} \bar{d}_{v, i j}
$$

Numerical investigation showed that

$$
\left\langle\bar{d}_{v, i i}^{\times}\right\rangle_{\phi}=\left\langle\bar{d}_{v, i i}\right\rangle_{\phi}
$$
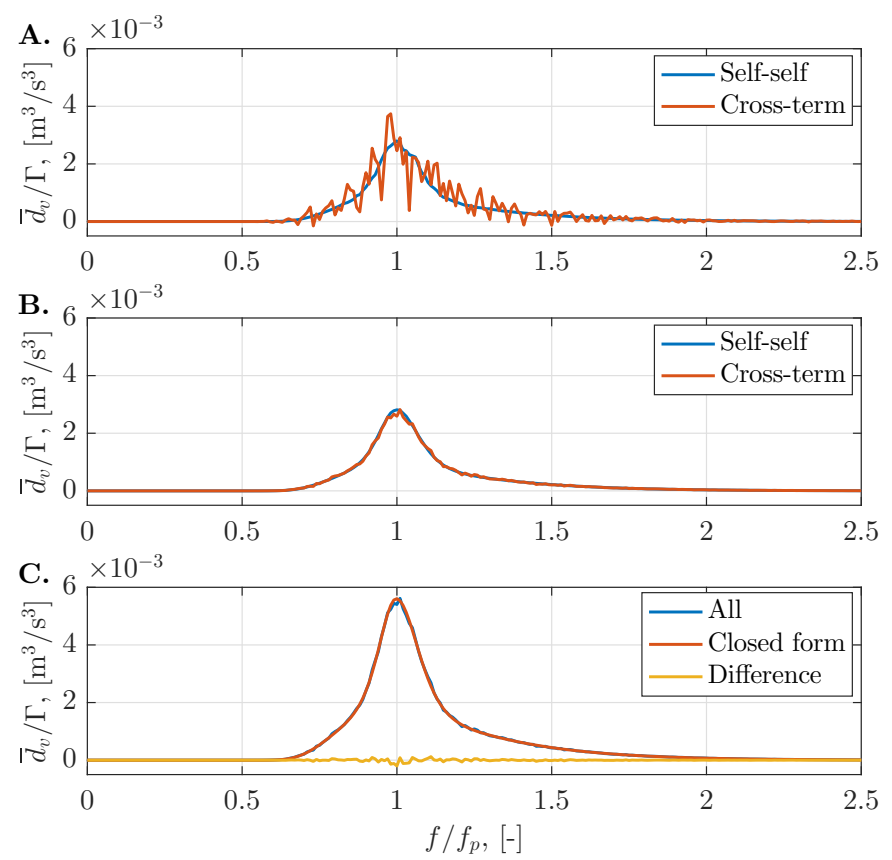

Figure 3: The frequency distribution of the dissipation terms. For $N_{w}=100$, $T_{p}=5 \mathrm{~s}, \gamma=3.3, h=2 \mathrm{~m}$ and $z / h=-1$. A: Self-self and cross-terms for one realisation of the random phases. B: Self-self and cross-terms averaged over 100 realisations of the random phases. C: Sum of self-self and cross-term averaged over 100 realisation, the closed form solution (Eq. (25)), and the difference between the two.

There is a substantial amount of scatter for each realisation of the random phases (Figure 3A), but the equality holds for an average over a number of realisations (Figure 3B). An extensive investigation showed the same behaviour for different wave periods, different water depths, vertical positions, and spectral shape, as long as more than 50 realisations are included and the duration of the time series exceeds $150-200$ peak wave periods. This finally leads to the following description of the bulk 
dissipation for the $i$ 'th frequency components at a given height $z$

$$
\bar{d}_{v, i}=\Gamma u_{m, i}^{2} \overline{\left|\sum\right|}=2 \Gamma S_{u, i} \Delta f_{i} \sqrt{\frac{2 m_{u, 0}}{\pi}}
$$

The spectral distribution of the dissipation is

$$
\bar{\delta}_{v}=2 \Gamma S_{u} \sqrt{\frac{2 m_{u, 0}}{\pi}}
$$

From Eqs. (25) and (26), the bulk dissipation at a given level is given as

$$
\bar{d}_{v}(z)=\sum_{i} \bar{d}_{v, i}=\int_{0}^{\infty} \bar{\delta}_{v} \mathrm{~d} f
$$

\subsection{Depth-integrated and bulk dissipations}

From Eq. (26) it follows that the depth-integrated frequency dependent dissipation can be described as

$$
\overline{\mathcal{D}}_{v}(f)=\int_{-h}^{-h+h_{v}} \bar{\delta}_{v} \mathrm{~d} z
$$

No closed form of Eq. (28) is identified, so numerical integration over the height of the vegetation is required. The Simpson's integration rule is applied and the required number of integration points is presented in Appendix A. A total of 501 integration points is applied throughout this work, which is much larger number than the minimal requirement.

The bulk dissipation is calculated as

$$
\overline{\mathcal{D}}_{v, t o t}=\int_{0}^{\infty} \overline{\mathcal{D}}_{v}(f) \mathrm{d} f
$$

The bulk dissipation quantity from Eq. (29) is comparable to $\overline{\mathcal{D}}_{M L}$ and $\overline{\mathcal{D}}_{\text {tot }}$ for irregular waves.

\section{Comparison of dissipation models}

In this section, dissipation values from the present model are quantitatively compared to those by Mendez and Losada (2004) (Section 3.1) and Suzuki et al. (2012) (Section 3.2). Next, an intercomparison between the two prior models is presented (Section 3.3) with an emphasis on explaining the verification presented in Suzuki et al. (2012). In the present work $\alpha_{u}=1$ is used to be consistent with the existing two models.

\subsection{Comparison with Mendez and Losada (2004)}

$\overline{\mathcal{D}}_{M L}$ is based on the assumptions that the spectrum is narrow banded and that the representative shape of the velocity profile is uniquely described by a representative period; taken as the peak wave period.
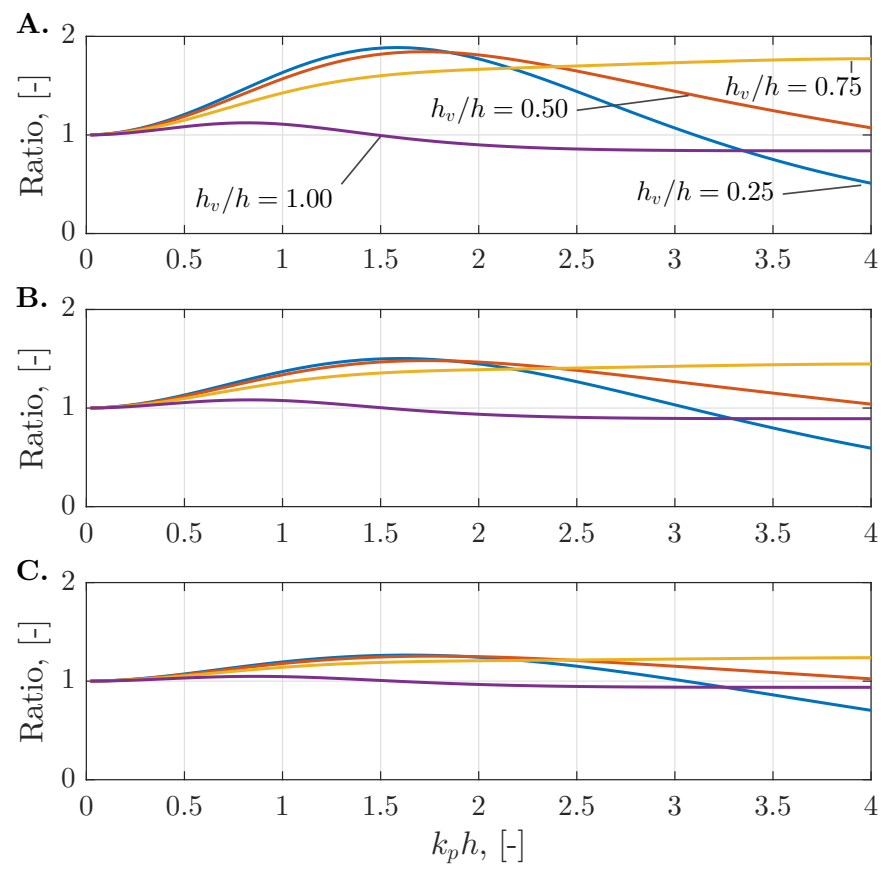

Figure 4: The ratio of the bulk dissipation $\overline{\mathcal{D}}_{M L} / \overline{\mathcal{D}}_{v, \text { tot }}$ as a function of $\gamma$ and the height of vegetation. A: $\gamma=1.0$. B: $\gamma=3.3$. C: $\gamma=10.0$.

The ratio $\overline{\mathcal{D}}_{M L} / \overline{\mathcal{D}}_{v, \text { tot }}$ is presented in Figure 4 as a function of the nondimensional wave number $k_{p} h$ for three values of the peak enhancement factor for the JONSWAP wave spectrum: $\gamma=\{1.0,3.3,10.0\}$. The ratio is furthermore evaluated for different relative vegetation heights $\left(h_{v} / h\right)$.

The ratio is unity for shallow water $\left(k_{p} h \ll 1\right)$, because the velocity profile is effectively uniform over the height for all frequency components, so the possible choices of a representative velocity profile have no effect. However, there are observed marked differences in the bulk dissipation for increasing values of $k_{p} h$. It is also observed that the discrepancy between the two dissipation models decreases for increasing $\gamma$, which is attributed to a narrowing of energy containing wave numbers. The effect of the choice of dissipation model is the smallest for emerging vegetation.

The present model is not restricted to the narrow-banded Rayleigh probability distribution and is expected to deliver a more accurate description of the dissipation due to vegetation because the dissipation cut-off frequency and true (to first-order) velocity profile are accounted. These improvements are particularly advantageous in scenarios with submerged canopies and broad wave energy spectra distribution in the intermediate to deep-water wave regime. The ratio plotted in Figure 4 eliminates the effects of the drag coefficient, thus the drag coefficient cannot be freely interchanged between models without an additional scaling factor. The additional scaling factor means that a fit between the dissipation model by Mendez and Losada (2004) and experimental data will result in drag coefficients, which are not only dependent on resistance properties of the stem, but also the degree of submergence, the wave period and the spectral shape. This may be one explanation for the large number of empirical expressions to calculate the 
drag coefficient found in the literature (Cavallaro et al., 2010; Jadhav et al., 2013; Ozeren et al., 2014; Anderson and Smith, 2014; Wu and Cox, 2015; Losada et al., 2016, among others). It further suggests that a re-analysis of the existing experimental data sets for irregular waves could be useful, while the regular wave sets are not likely to provide additional insights since ratio $\overline{\mathcal{D}}_{M L} / \overline{\mathcal{D}}_{v, \text { tot }}$ goes to 1.0 for large peak enhancement factors.

\subsection{Comparison with Suzuki et al. (2012)}

\subsubsection{Bulk dissipation}

Figure 5 shows a comparison of the bulk dissipation by Suzuki et al. (2012) and the present work through the ratio $\overline{\mathcal{D}}_{S W A N} / \overline{\mathcal{D}}_{v, \text { tot }}$. The following main observations are made: (i) The maximum in the ratio is smaller than in Figure 4. (ii) The ratio drops faster for increasing $k_{p} h$ in comparison to the dissipation model by Mendez and Losada (2004). (iii) The ratio does not tend to unity for small values of $k_{p} h$.
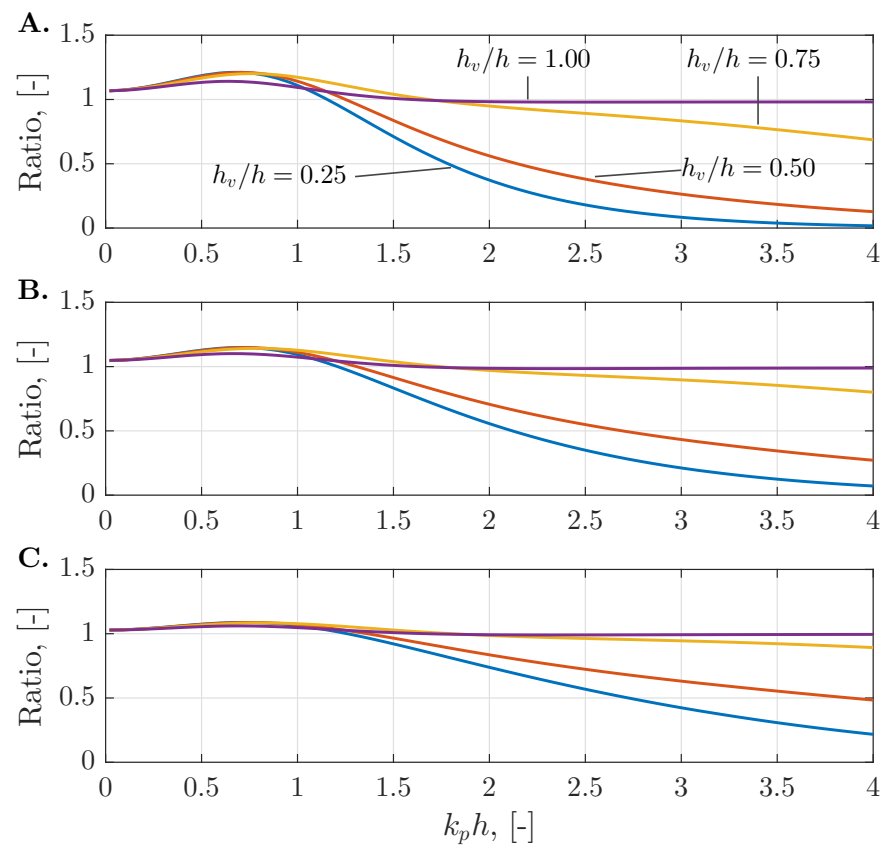

Figure 5: The ratio of the bulk dissipation $\overline{\mathcal{D}}_{S W A N} / \overline{\mathcal{D}}_{v, \text { tot }}$ as a function of $\gamma$ and the height of vegetation. A: $\gamma=1.0$. B: $\gamma=3.3$. C: $\gamma=10.0$.

The smaller maximum value noted in observation (i) is likely explained by the fact that $\omega_{m 0}$ is representative for the zerocrossing wave period and hence a good representative frequency in shallower water and/or large values of $h_{v} / h$. Observation (ii) is explained by the fact that the representative frequency and wave number are based on a spectral average $\left(\omega_{m 0}\right.$ and $k_{m 0}$ ), where $\omega_{p}<\omega_{m 0}$, so the velocity profile exhibits a more rapid decay towards the bed than is the case for $\omega_{p}$.

Observation (iii) is also related to the spectral definition of $k_{m 0}$ and $\omega_{m 0}$ and the fact that the set $\left\{k_{m 0}, \omega_{m 0}\right\}$ does not fulfil the linear dispersion relation. The consequence is that the shape function linking $S_{\eta}$ and $S_{u}$ is not based on linear wave theory and thus represents a fundamental mismatch in the theories between Suzuki et al. (2012) and the present work (as well as Mendez and Losada, 2004). The discrepancy is $7 \%$ for $\gamma=1.0$,
$5 \%$ for $\gamma=3.3$, and $3 \%$ for $\gamma=10.0$ in the limit of small $k_{p} h$. A modified cyclic frequency, $\tilde{\omega}_{m 0}$, fulfilling the linear dispersion relation with $k_{m 0}$ (from Eq. 5) yielded a ratio of 1.0 for small values of $k_{p} h$.

The analysis shows that the dissipation model by Suzuki et al. (2012) contains much less variability in the deviation from the present work with the vegetation height for small values of $k_{p} h$. The present analysis suggests the dissipation model by Suzuki et al. (2012) is generally adequate when $k_{p} h<1.0$ and $3.3 \leq$ $\gamma$. Replacing $\omega_{m 0}$ with $\tilde{\omega}_{m 0}$ would improve the fitting between Suzuki et al. (2012) and the present work. For larger values of $k_{p} h$ a considerable under-prediction of the energy dissipation is to be expected unless special tuning of the drag coefficient is included. Examples of corresponding water depth and period limits are presented in Table 1 . These recommendations apply to the bulk dissipation and do not extend to the dissipation on individual frequencies, which is discussed below.

Table 1: The correspondence between water depth and minimum allowed peak wave period in order to fulfil $k_{p} h<1.0$.

\begin{tabular}{c|cc|cccccc}
$\max k_{p} h$ & $h$ & {$[\mathrm{~m}]$} & 0.5 & 1.0 & 1.5 & 2.0 & 3.0 & 5.0 \\
\hline 0.7 & $\min T_{p}$ & {$[\mathrm{~s}]$} & 2.2 & 3.1 & 3.8 & 4.4 & 5.3 & 6.9 \\
1.0 & $\min T_{p}$ & {$[\mathrm{~s}]$} & 1.6 & 2.3 & 2.8 & 3.3 & 4.0 & 5.1
\end{tabular}

\subsubsection{Frequency distribution of the dissipation}

The frequency distribution of the dissipation for the models by Suzuki et al. (2012) and the present work is presented for $h_{v} / h=0.5$ with two values of $\gamma$ and four values of $k_{p} h$ in Figure 6.

The characteristic differences between the two models clearly show: (i) There is a cross-over point adjacent to the mean wave period, where the dissipation is the same for the two models. (ii) The smaller $k_{p} h$, the smaller the difference between the two models. (iii) The maximum dissipation by the Suzuki et al. (2012) model is always located at the peak frequency, $f_{p}$, whereas the inclusion of the individual velocity profiles for each frequency within the present model causes the peak of the dissipation to shift to frequencies lower than $f_{p}$. This is particularly clear for $\gamma=1.0$ and $k_{p} h$ of 1.5 and 3.0. (iv) The dissipation is greatly underestimated by the Suzuki et al. (2012) model for frequencies lower than $f_{p}$ in deeper water $\left(1<k_{p} h\right)$.

The peak in dissipation for $f<f_{p}$ and large $k_{p} h$ seems to have gone unnoticed in the literature. The peak in dissipation is close to $f_{p}$ for $\gamma=3.3$ and all $k_{p} h$ and $\gamma=3.3$ is a common value used in laboratory experiments (Ma et al., 2013; Méndez et al., 1999; Möller et al., 2014; Wu et al., 2016; SánchezGonzález et al., 2011). Field campaigns reporting (or indicating) peak dissipation at $f_{p}$ are typically for $k_{p} h<1$ (Jadhav et al., 2013; Lowe et al., 2007) and the present theory indeed suggests the peak in dissipation to occur for $f=f_{p}$ for $k_{p} h<1$ (Figure 6A-B). 

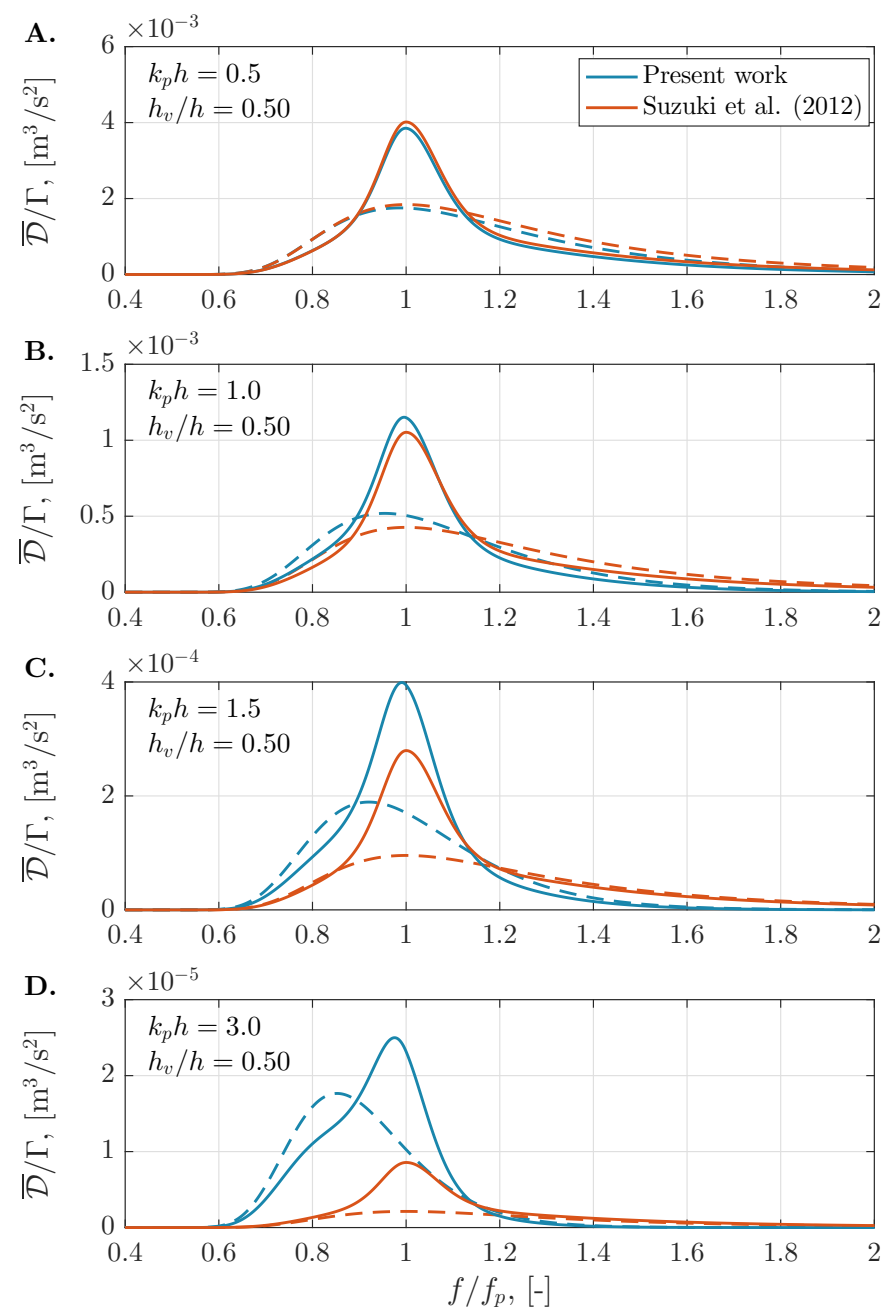

Figure 6: The frequency-distribution of the energy dissipation for different values of $k_{p} h$ for $\gamma=1.0$ (dashed lines) and $\gamma=3.3$ (full lines). A: $k_{p} h=0.5$. B: $k_{p} h=1.0$. C: $k_{p} h=1.5$. D: $k_{p} h=3.0$.

\subsection{Verification in Suzuki et al. (2012)}

Suzuki et al. (2012) presented verification of their frequency distributed dissipation model against the model by Mendez and Losada (2004). A perfect match between the two dissipation models is presented (Suzuki et al., 2012, their Figure 2) with an academic case for a range of $k_{p} h \in[0.29,8.1]$. The perfect match is surprising given the widely different bulk dissipation presented in Figures 4 and 5. The explanation is found in the fact that the comparison was performed for a value of the spectral width parameter

$$
v=\frac{m_{0} m_{2}}{m_{1}^{2}}-1=0.01
$$

where $m_{0}, m_{1}$ and $m_{2}$ are the zeroth, first, and second order moments of $S_{\eta}$. The value of 0.01 represents a narrow spectrum, which is close to a regular wave with $\gamma \approx 300$ in the case of a JONSWAP spectrum. Consequently, $\omega_{p} \simeq \omega_{m 0}$ and $k_{p} \simeq k_{m 0}$ and the representative velocity profiles becomes close to iden- tical. $^{2}$

The second verification is an application of the two dissipation models to predict the wave attenuation as measured in the experimental setup by Lovas (2000) (see Suzuki et al., 2012, their Figure 4). Both dissipation models result in qualitatively identical results and a good validation for identical resistance properties of the vegetation $\left(N, w_{v}, h_{v}\right.$, and $\left.C_{D}\right)$ for two values of $T_{p}: 2.5 \mathrm{~s}$ and $3.5 \mathrm{~s}$. At the offshore edge of the vegetation this corresponds to $k_{p} h=0.60$ and $k_{p} h=0.42$ and these intermediate values of $k_{p} h$ explains the close match, because the ratio of $\overline{\mathcal{D}}_{M L}$ over $\overline{\mathcal{D}}_{S W A N}$ is within $\pm 2 \%$ for the applied peak enhancement factor of $\gamma=7.0$ and the given values of $k_{p} h$.

\subsection{Discussion of drag coefficients}

Several experimental campaigns with irregular waves have applied the dissipation model from Mendez and Losada (2004) to wave attenuation measurements in order to calculate a bulk drag coefficient (Cavallaro et al., 2010; Ozeren et al., 2014; Anderson and Smith, 2014; Losada et al., 2016, among others). The results in Figure 4 show that the resulting drag coefficients based on the Mendez and Losada (2004) model will be heavily influenced by the spectral shape and height of the vegetation. The Rayleigh distribution (narrow-band) assumption of the Mendez and Losada (2004) model may be violated for irregular waves with a broad spectrum such as storm waves (Chen and Zhao, 2012).

Additionally, it has been shown that bulk drag coefficients derived from the Mendez and Losada (2004) model cannot be universally applied to an energy spectral dissipation model such as Suzuki et al. (2012) or the present model. A comparison of the dissipation models from Mendez and Losada (2004) and Suzuki et al. (2012) is shown in Figure 7 as a function of $k_{p} h$ for the JONSWAP wave spectrum with three values of the peak enhancement factors: $\gamma=1.0,3.3,10.0$. To obtain the same bulk dissipation at $k_{p} h=1.5$, the drag coefficients between Mendez and Losada (2004) and Suzuki et al. (2012) may have to be as much as a factor of 3 different.

An appropriate limit to use drag coefficients based on Mendez and Losada (2004) in conjunction with Suzuki et al. (2012) seems to be $k_{p} h<0.7$ and $\gamma \geq 1.0$. This yields discrepancies between the two models of less than $15 \%$. The $15 \%$ error threshold corresponds to the maximum discrepancy between Suzuki et al. (2012) and the present work for $k_{p} h<1$ and $\gamma \geq 3.3$.

\section{Validation}

\subsection{Experimental setup}

An experimental campaign was conducted at the University of Aberdeen in a $20 \mathrm{~m}$ long, $0.45 \mathrm{~m}$ wide wave flume operating at water depth of $0.7 \mathrm{~m}$ (Figure 8). Waves were generated by

\footnotetext{
${ }^{2}$ Note that the authors assumed that Suzuki et al. (2012) used the spectral width definition shown in Eq. 30 and not $\sqrt{v}$. The latter results in $\gamma>10,000$ for a JONSWAP spectrum.
} 

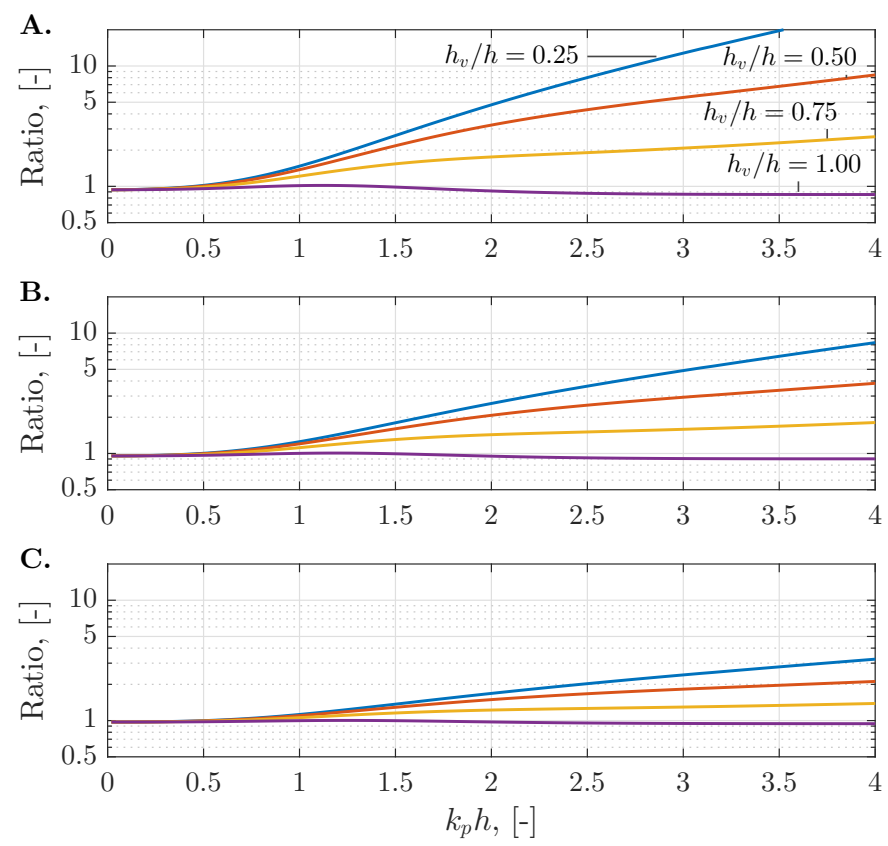

Figure 7: The ratio of the bulk dissipation $\overline{\mathcal{D}}_{M L} / \overline{\mathcal{D}}_{S W A N}$ as a function of $\gamma$ and the height of vegetation. A: $\gamma=1.0$. B: $\gamma=3.3$. C: $\gamma=10.0$.

a single-flap bottom-hinged wave paddle with active wave absorption while at the other end of the flume a porous parabolicshaped beach was installed to dissipate the waves.

The artificial vegetation elements consisted of $4 \mathrm{~mm}$ wide polypropylene blades of varying length and thicknesses, four of which were taped to a $75 \mathrm{~mm}$ long and $6 \mathrm{~mm}$ diameter PVC dowel (stem). The dowels were inserted in a staggered arrangement in a $15 \mathrm{~mm}$ thick PVC false floor placed on the bottom of the flume and protruded by $60 \mathrm{~mm}$ above the bed in a staggered configuration (see sketch in Figure 8B-C). The length of the canopy was $7.5 \mathrm{~m}$, with a vegetation density of 566 dowels $/ \mathrm{m}^{2}$. Due to the raised false floor the water depth in the vegetation canopy was $0.685 \mathrm{~m}$. To study the role of vegetation height, three vegetation heights, $h_{v}$, were tested with a blade thickness of $0.5 \mathrm{~mm}$ corresponding to relative submergence $h_{v} / h=\{0.38,0.67,0.96\}$. To study the role of vegetation flexibility, four different vegetation flexibilities were tested at the $h_{v} / h=0.38$ submergence. The flexibility was controlled by the blade thickness, which was tested with thicknesses of $0.12,0.2,0.5,1.0 \mathrm{~mm}$, which ranges from highly flexible vegetation to semi-rigid. The cases shown in this manuscript are a small subset of a much larger experimental campaign focused on wave-induced hydro- and turbulence dynamics within flexible vegetated canopies that will be more thoroughly documented in subsequent manuscripts.

The water surface elevation was measured with six twin-wire resistance-type wave gauges, four of the gauges were moved during repeat trials to measure the water surface elevation at a total of 10 locations. The wave gauges WG1 and WG2 were fixed during repeats. The wave conditions involved two irregular wave time-series each based a single peaked JONSWAP spectrum with a peak enhancement factor $\gamma=3.3$ and peak wave period $T_{p}=1.15 \mathrm{~s}$ with two different wave heights at the beginning of the vegetation $(x=0 \mathrm{~m})$ of $H_{m 0}=6.8 \mathrm{~cm}$ and 3.7 $\mathrm{cm}$. The measurement duration of each experimental run was $1200 \mathrm{~s}$. Note that for each wave condition reference measurements without vegetation were carried out in order to determine the dissipation resulting from skin friction along the glass sidewalls and flume (false) floor.

\subsection{Validation of shape of the dissipation spectrum}

The wave spectra for the vegetated, $S_{\eta}^{v}$, and reference cases, $S_{\eta}^{r}$, were calculated for all wave gauges. Based on these, the energy dissipation due to vegetation could be approximated:

$$
\frac{\partial c_{g} S_{\eta}}{\partial x} \simeq \frac{\Delta S_{\eta}^{v}-\Delta S_{\eta}^{r}}{\Delta x}
$$

where $\Delta S_{\eta}$ is the difference over the distance $\Delta x$ within the canopy. The two wave gauges at the offshore and onshore boundaries of the canopy (WG3 and WG6) are used in the evaluation of Eq. (31). The measured spectral shape can then be compared with the average of the dissipation as calculated according to the new method (Eq. (28)), where the measured surface elevation was used. However, given that neither the drag coefficient nor the velocity reduction is known, the normalised spectra are depicted in Figure 9A. Note that all spectra in Figure 9A are normalised with their respective peak value. The spectral shape between the measured and theoretical predictions are seen to be almost identical, though possibly with a weak overprediction of the dissipation below the peak frequency $(0.87 \mathrm{~Hz})$. This overprediction is so limited that it cannot be stated, whether it is due to accuracy of the experiments or has a physical explanation. In the same figure, the predicted shape of the dissipation by Suzuki et al. (2012) is considerably different from the experimental and present model shapes. The shape differences match the theoretical prediction discussed in conjunction with Figure 6: an underpredicted normalised dissipation for $f<f_{p}$ and an overpredicted normalised dissipation for $f_{p}<f$.

\subsection{Comparison with Suzuki et al. (2012)}

Following the validation of the spectral shape of the dissipation based on the new method, a comparison between the theoretical dissipation using the present work (Eq. 28) and Suzuki et al. (2012) (Eq. (3)) using the measured wave spectrum, $S_{\eta}$, is presented. This comparison is depicted in Figure 10 for the case with vegetation blade thickness of $1 \mathrm{~mm}$ and vegetation submergence of $h_{v} / h=0.38$ at two wave gauges within the canopy. It can be seen that the present work predicts larger dissipation in the peak frequency range, which corroborates the results shown in Figure 5B, and the present model does not dissipate any energy at frequencies higher than a certain frequency cut-off. The frequency cut-off is defined as the frequency at which the wave orbital velocities at top of the canopy vanishes, thus no energy will be dissipated by the submerged vegetation. This frequency cut-off is given by linear wave theory as 
A.

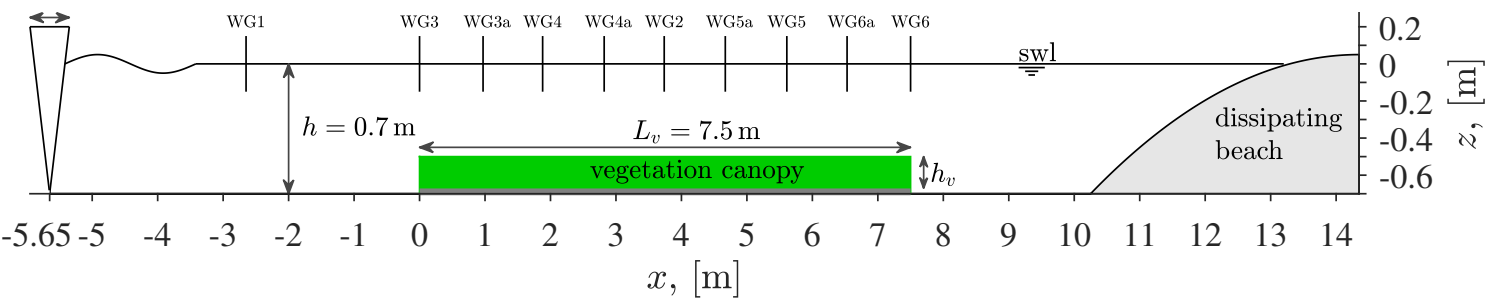

B.

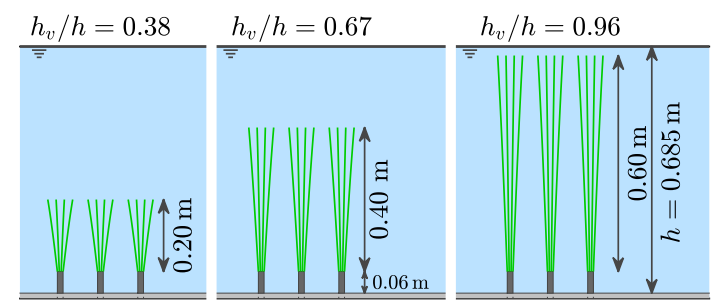

C.

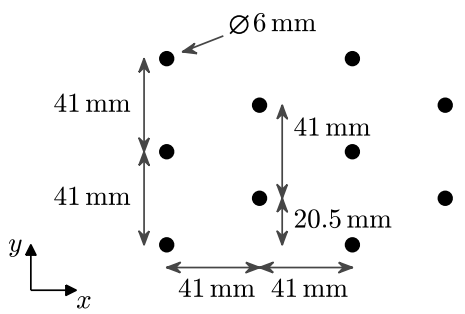

Figure 8: A: Flume layout of the physical model. Wave gauge measurement locations are labeled and shown with vertical solid lines, and the label "a" notes the gauge's measurement position during repeat trials. B: Sketch of the vegetation mimics for three heights. C: Planar view of the staggered installation of the mimics.

$$
f_{\text {cut }-o f f}^{2}=\frac{1}{2\left(h-h_{v}\right)} \frac{g}{2 \pi}
$$

The frequency cut-off is shown in Figure 10.

The ratio of the bulk dissipation at each wave gauge using SWAN's Suzuki model compared to the new model for all four vegetation flexibilities was in the range: $0.74 \leq$ $\overline{\mathcal{D}}_{S W A N} / \overline{\mathcal{D}}_{v, \text { tot }} \leq 0.87$. The vegetation flexibility is not relevant in this ratio as it is accounted for in the $\Gamma$ parameter which is divided out. The smaller ratio values (larger difference between the models) occurred for the smaller wave height $\left(H_{m 0}=3.7\right.$ $\mathrm{cm})$.

\subsection{Frequency dependent decay}

The frequency cut-off of the dissipation is investigated based on normalised spectra:

$$
S_{\eta}^{*}=\frac{S_{\eta}}{S_{\eta, x=0}}=\frac{1}{\left(1+\beta_{f} x\right)^{2}}
$$

where the rightmost expression is an ad hoc extension of Eq. (8) with a frequency dependent decay factor $\beta_{f}$. The normalised spectra are adopted to emphasize the rate of decay over the spectral energy distribution. The gradient in $S_{\eta}^{*}$ thus follows as:

$$
\frac{\partial S_{\eta}^{*}}{\partial x} \simeq-2 \beta_{f}+O\left(\beta_{f}^{2}\right)
$$

where the right-hand side is the Taylor expansion to leading order. The experimental shape of $-2 \beta_{f}$ is shown in Figure 9B along with the theoretical shape. The theoretical shape follows from the wave energy conservation equation (e.g. Smit et al., 2012; Van Rooijen et al., 2016):

$$
\frac{\partial c_{g} S_{\eta}}{\partial x}=c_{g} \frac{\partial S_{\eta}}{\partial x}=-\overline{\mathcal{D}}
$$

where $c_{g}$ is the group velocity, which is assumed indedependent of $x$. Reorganising Eq. (35) and normalising by $S_{\eta, x=0}$ it follows that

$$
\frac{1}{S_{\eta, x=0}} \frac{\partial S_{\eta}}{\partial x}=-\frac{\overline{\mathcal{D}}}{c_{g} S_{\eta, x=0}} \simeq-2 \beta_{f}
$$

This relationship is used, when comparing the present model and Suzuki et al. (2012) with experimental results in Figure 9B, with $\Gamma / \rho=11$ obtained by fitting. The results clearly show that the present method predicts the spectral shape of $-2 \beta_{f}$, while Suzuki et al. (2012) does not. The non-uniformity of $\beta_{f}$ for Suzuki et al. (2012) follows from the increase in $c_{g}$ with decreasing $f$.

Figure 11 depicts $-2 \beta_{f}$ as a function of frequency for 4 values of the blade thickness. Recall that $-2 \beta_{f}$ is a relative decay and the dimensional spectral gradient reads:

$$
\frac{\partial S_{\eta}}{\partial x} \simeq-2 \beta_{f} S_{\eta, x=0}
$$

In Figure 11, the normalised spectral gradients for the vegetated case and the reference case are different for lower frequencies and converge with increasing frequency. By subtracting the reference measurements from the vegetated measurements, the difference line shows the normalised spectral gradient caused by the dissipation due to vegetation. The role of the vegetation flexibility can be seen in the difference lines: dissipation increases with increasing blade thickness. Wave reflections in the flume are the likely cause of the oscillations in the vegetation and reference measuremnts. The difference line can be seen 

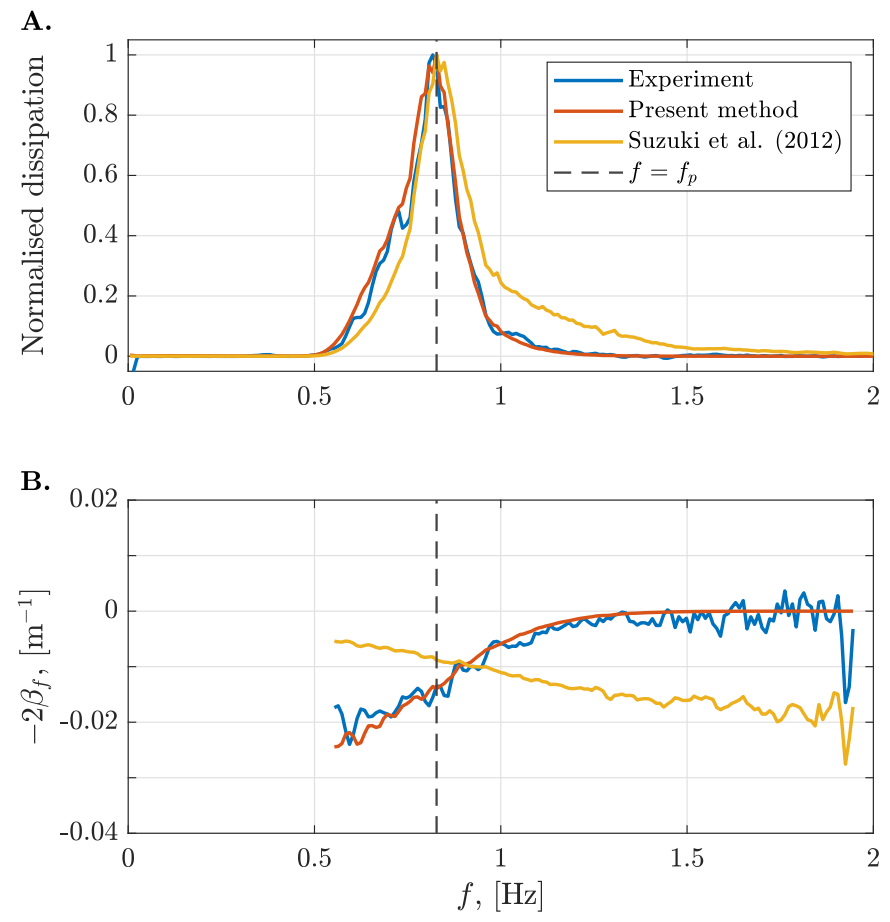

Figure 9: A: Comparison between the measured, normalised dissipation spectrum (Eq. 31) and the theoretical prediction of the normalised dissipation spectrum (Eq. 28). $N=566 \mathrm{stems} / \mathrm{m}^{2}$, vegetation blade thickness of $1 \mathrm{~mm}$, $h_{v} / h=0.38, H_{m 0} / h=0.054, T_{p}=1.15 \mathrm{~s}$, and $k_{p} h=2.1$. B: The corresponding experimental and theoretical variation of $-2 \beta_{f}$.

converging to zero for frequencies smaller than the frequency cut-off for all four vegetation flexibilities, matching the results of the present work.

The difference in the normalized spectral gradient between the vegetated and non-vegetated (reference) cases are shown for the three submergence ratios tested in Figure 12. The difference lines in Figure 12 converge to zero at or at a lower frequency than their respective frequency cut-off for the two shorter vegetation heights, matching the results of the present work. The tallest vegetation fills the majority of the water column and the normalised spectral gradient difference does not converge to zero since all the energy in the wave spectrum is affected by the vegetation. The comparison with the present theoretical model is shown as markers following Eq. (36), where $\Gamma / \rho=\{8.5,5.2,1.8\}$ is used for $h_{v} / h=\{0.38,0.67,0.96\}$, respectively. It is seen that the relative decay $-2 \beta_{f}$ is more rapid for low frequencies than high frequencies for $h_{v} / h=0.38$ and the opposite is found for $h_{v} / h=0.96$. The explanation is found in the wave number effect and the velocity transformation function Eq. (9), which favours low frequency waves for large submergence. For near-emergent vegetation, the velocity transformation function has limited effect (since the present method converges to that by Suzuki et al. (2012)), so the more rapid decay at high frequencies is explained by the increase in $c_{g}$ with decreasing $f$, since $c_{g}$ is in the denominator in Eq. (36). The values of $\beta_{f}=-2 \beta_{f} /(-2)$ up to $0.025 \mathrm{~m}^{-1}$ is observed for a spectral wave height of $0.04 \mathrm{~m}$, which is a smaller value than reported values in Maza et al. (2015), their figure 7, who found
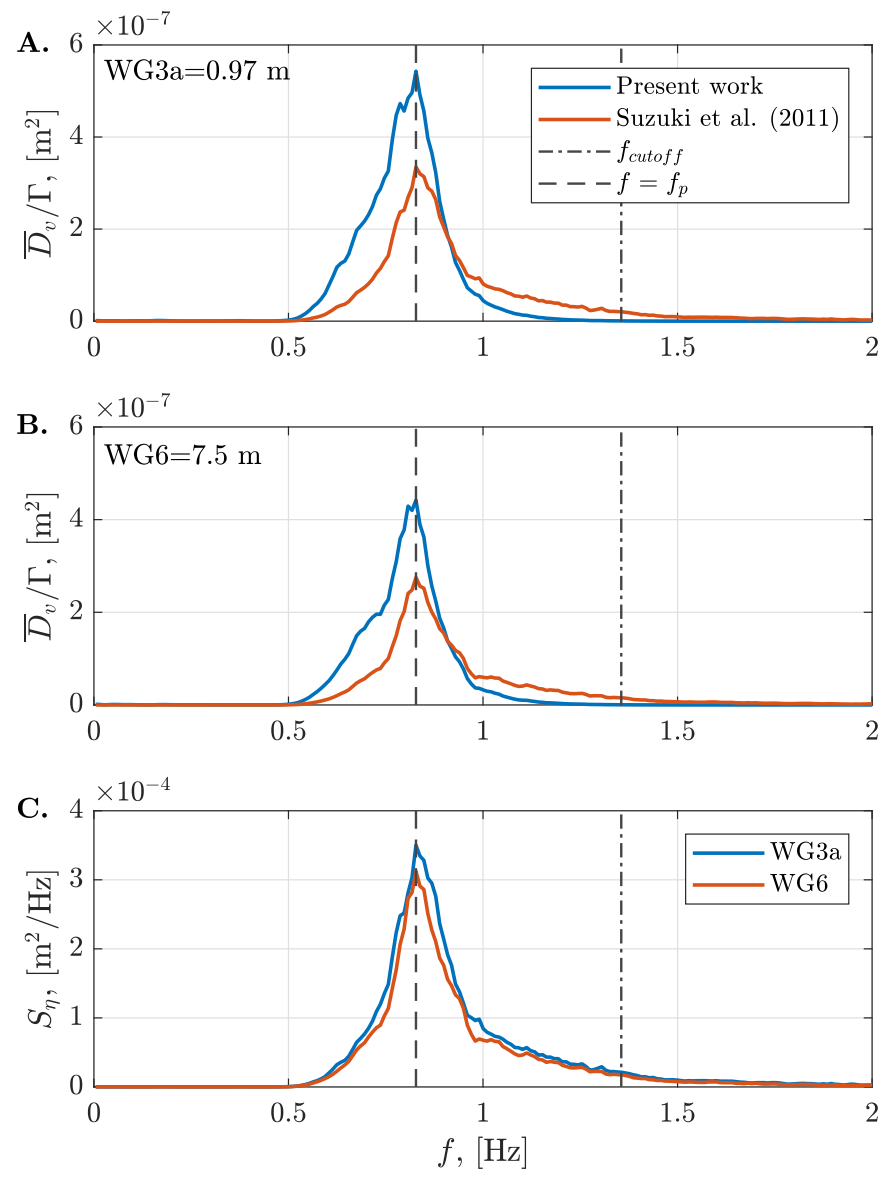

Figure 10: The dissipation at wave gauge 3a (A) and wave gauge 6 (B) using the new dissipation model and the Suzuki et al. (2012) model. The measured wave spectra at both gauges (C) in the vegetation field with $N=566 \mathrm{stems} / \mathrm{m}^{2}$, vegetation blade thickness of $1 \mathrm{~mm}, h_{v} / h=0.38, H_{m 0} / h=0.054, T_{p}=1.15 \mathrm{~s}$, and $k_{p} h=2.1$.

$\beta^{\prime}$ between $0.055 \mathrm{~m}^{-1}$ and $0.175 \mathrm{~m}^{-1}$ for irregular waves in 0.6 $\mathrm{m}$ and $0.4 \mathrm{~m}$ of water depth, respectively, with a spectral wave height of $0.12 \mathrm{~m}$. Note that there is a difference between the bulk $\left(\beta^{\prime}\right)$ and frequency-varying $\left(\beta_{f}\right)$ decay factors. The magnitude difference is ascribed to the larger wave height in Maza et al. (2015).

\subsection{Relating $\Gamma / \rho$ to $C_{D} \alpha_{u}^{3}$}

The relationship between $\Gamma / \rho$ and $C_{D} \alpha_{u}^{3}$ from Section 2.2 reads:

$$
\Gamma / \rho=\frac{1}{2} \tilde{N} \tilde{b}_{v} C_{D} \alpha_{u}^{3}
$$

where $\tilde{N}$ and $\tilde{b}_{v}$ are stem averaged quantities given the fact that the stems are not constant over the full canopy height. The depth-averaged product is estimated as

$$
\tilde{N} \tilde{b}_{v}=\frac{1}{h_{v, 1}+h_{v, 2}}\left(N_{1} b_{v, 1} h_{v, 1}+N_{2} b_{v, 2} h_{v, 2}\right)
$$



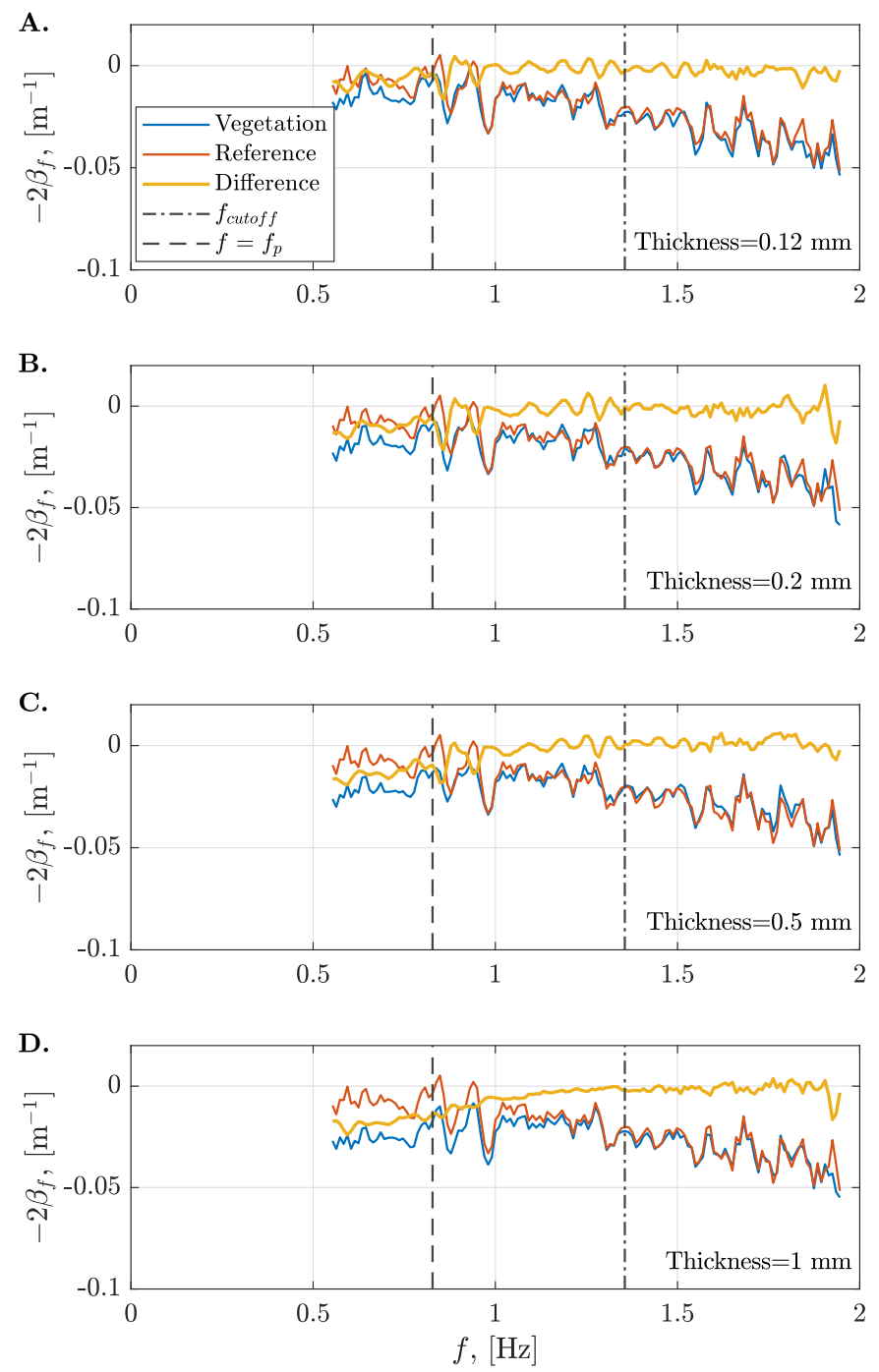

Figure 11: The measured normalized spectral gradient through the vegetation, without vegetation (reference), and difference between the gradients for vegetation with blade thicknesses of 0.12 (A), $0.2(B), 0.5$ (C), and $1 \mathrm{~mm}$ (D) in the vegetation field with $N=566 \mathrm{stems} / \mathrm{m}^{2}, h_{v} / h=0.38, H_{m 0} / h=0.054$, $T_{p}=1.15 \mathrm{~s}$, and $k_{p} h=2.1$. The frequency cut-off is shown with the vertical dashed line.

where subscripts 1 and 2 refer to the dowels and flexible blades, respectively.

A constant value of $\Gamma$ gave a fairly accurate fit to the measured decay factor $-2 \beta_{f}$ despites the spectral noise. It suggests that $C_{D} \alpha_{u}^{3}$ is fairly frequency-independent for the present experimental setup. $C_{D}$ itself could not be determined in this work, since the velocity reduction $\alpha_{u}$ was not measured. $C_{D} \alpha_{u}^{3}$ can be directly evaluated by combining Eqs. (38) and (39) with fitted values of $\Gamma / \rho . C_{D} \alpha_{u}^{3}=2.8$ for the case in Figure $9 \mathrm{~B}$, and the values corresponding to Figure 12 are $C_{D} \alpha_{u}^{3}=\{2.2,1.3,0.4\}$ for $h_{v} / h=\{0.38,0.67,0.96\}$, respectively. These values can be compared with bulk drag coefficients in the literature, which ranges from 0.4 (Méndez et al., 1999) to 10 Ozeren et al. (2014) for irregular waves, however, a direct comparison should be treated carefully, since the referenced works did not apply the dissipation method presented in this work.

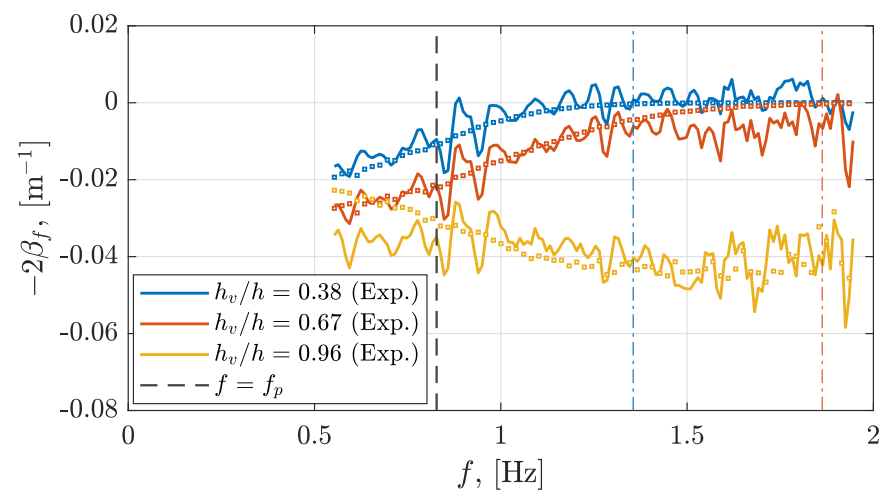

Figure 12: The measured difference between the normalized spectral gradient through the vegetation and without vegetation for vegetation submergences of $h_{v} / h=\{0.38,0.67,0.96\}$ with a vegetation field with $N=566 \mathrm{stems} / \mathrm{m}^{2}$, $H_{m 0} / h=0.054, T_{p}=1.15 \mathrm{~s}$, and $k_{p} h=2.1$. The frequency cut-off for the vegetation submergences of $h_{v} / h=0.38$ and 0.67 are shown with the coloured vertical dashed-dotted lines. The frequency cut-off for the submergence of $h_{v} / h=$ 0.96 was much higher than the wave energy tested. Coloured markers show the theoretical variation of $-2 \beta_{f}$ following the present dissipation method following Eq. (36).

\section{Discussion}

\subsection{Effect of directional spreading}

The effect of directional spreading on the bulk energy dissipation (in a given layer) is discussed here. It is intended as a first investigation of whether there are additional corrections required, when considering a directionally spread spectrum in comparison to the uni-directional spectrum detailed in Section 2.

The directional spreading means that the horizontal velocity field becomes a vector $\mathbf{u}=\{u, v\}$ and the dissipation in a given layer reads:

$$
\bar{d}_{\theta, v}=\overline{\mathbf{F}_{v} \alpha_{u} \cdot \mathbf{u}}=\overline{\Gamma \mathbf{u} \cdot \mathbf{u}\|\mathbf{u}\|_{2}}
$$

where $\mathbf{F}_{v}=1 / 2 \rho N w C_{D} \alpha_{u}^{2} \mathbf{u}\|\mathbf{u}\|_{2}$ is the force vector in a given layer. $\bar{d}_{\theta, v}$ is the dissipation accounting for directional spreading. The directional spreading is described with the cosinespreading function:

$$
\Lambda^{*}=\cos ^{2 \beta} \frac{\theta-\theta_{m}}{2}
$$

and

$$
\Lambda=\Lambda^{*}\left(\int_{-\pi / 2+\theta_{m}}^{-\pi / 2+\theta_{m}} \Lambda^{*} \mathrm{~d} \theta\right)^{-1}
$$

where $\beta$ is a spreading factor, $\theta=\theta_{m}+[-\pi / 2, \pi / 2]$ is the wave direction, and $\theta_{m}$ is the mean wave direction. In the remainder, $\theta_{m}=0$. For each wave direction, the spectrum $S_{\eta}^{\prime}(\theta)=\Lambda(\theta) S_{\eta}$ is used to calculate the velocity vector for the given direction. The direction of the velocity vector follows directly from $\theta$. The total velocity vector comes from summing over all directions. 
The ratio $\bar{d}_{\theta, v} / \bar{d}_{v}$ for different values of $\beta$ is plotted in Figure 13. The Figure represents 18,000 realisations based on 36 combinations of $z, \gamma$, and $T_{p}$. It is seen that $\bar{d}_{\theta, v} / \bar{d}_{v}$ becomes 1.0 for large $\beta$ for which $\Lambda$ is effectively unidirectional. In the opposite end, where $\Lambda$ is a uniform distribution, $\bar{d}_{\theta, v} / \bar{d}_{v}=0.84$. As a reference, it is noted that common values of $2 \beta$ are in the range $2-10$, where $2-4$ is for sea waves and 10 for swell waves. The default values according to The SWAN Team (2013) is $2 \beta=2$.

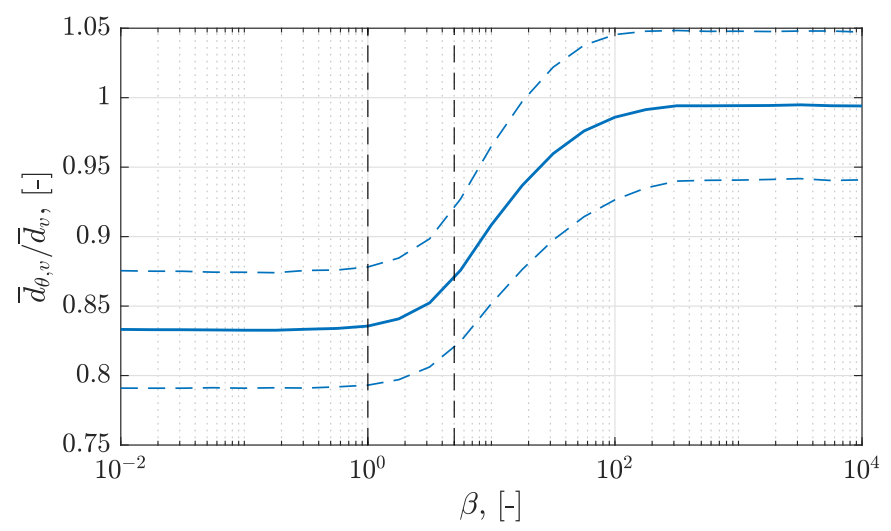

Figure 13: The ratio $\bar{d}_{\theta, v} / \bar{d}_{v}$ over $\beta$ with mean as full line and \pm standard deviation as dashed lines. Vertical black, dashed lines show common interval for $\beta$.

This preliminary analysis suggests that the directional spreading does influence the bulk dissipation and it is hypothesised that this is due to different random phases for the same frequency, but different directions. It is furthermore noted that $\Lambda$ is just one of several possible spreading distributions and it is anticipated that differences will arise between these. However, considering the extent of such an analysis, it is deemed outside the scope of the present work to derive detailed closure formulations of the distribution of the energy dissipation over both frequencies and directions for arbitrary directional spreading functions.

Until an in-depth analysis has been performed, the reduction in dissipation due to directional spreading as shown in Figure 13 can be used uniformly across the spectrum. Based on common values of $\beta$, it is suggested to apply a correction factor of 0.85 .

\subsection{Comparison with Madsen et al. (1988)}

The impact of the nonlinear approach to derive the dissipation (Section 2) can be compared directly to the dissipation due to bottom friction as presented in Madsen et al. (1988). Madsen et al. (1988), their Eq. (26), arrived at a linearised rate of energy dissipation reading:

$$
\overline{\mathcal{D}}_{b, M P G}=\left.\overline{\tau u}\right|_{z=-h}=\frac{1}{4} f_{w} \rho u_{b r} u_{m, i}^{2}
$$

Here, $\tau$ is the bed shear stress, $u$ is the free stream velocity given as Eq. (10), $f_{w}$ is a friction factor and $u_{b r}$ is a representative (and constant) nearbed velocity. Madsen et al. (1988) defined $\tau=1 / 2 \rho f_{w} u u_{b r}$, however, employing the more general form $\tau=1 / 2 \rho f_{w} u|u|$, Eq. (43) can be written as

$$
\overline{\mathcal{D}}_{b}=\left.\overline{\tau u}\right|_{z=-h}=2 \Gamma_{b} S_{u, i} \Delta f_{i} \sqrt{\frac{2 m_{u, 0}}{\pi}}=\Gamma_{b} u_{m, i^{2}} \sqrt{\frac{2 m_{u, 0}}{\pi}}
$$

following the notation proposed in the present work, where $\Gamma_{b}=1 / 2 \rho f_{w}$ for bottom friction. The ratio between the expressions in Eqs. (44) and (43) is:

$$
\frac{\overline{\mathcal{D}}_{b}}{\overline{\mathcal{D}}_{b, M P G}}=\frac{2 \sqrt{2 m_{u, 0}}}{\sqrt{\pi} u_{b r}}=\frac{2}{\sqrt{\pi}}=1.13
$$

Here, the second equality follows from the definition of $u_{b r}^{2}=$ $2 m_{u, 0}$ in Madsen et al. (1988), their Eq. (21). This shows that the two expressions are equivalent, but they do not match perfectly. The main cause of this discrepancy is found in the fact that Madsen et al. (1988) evaluated the friction with $u_{b r}$ as a constant scaling. This means that they can employ the orthogonality principal in averaging $\overline{u_{b r} u^{2}}=u_{b r} \overline{u^{2}}$; effectively the contribution from the cross-terms is ignored. The omission of the cross-terms introduces a difference of a factor of 2 , while the lack of $1 / \sqrt{\pi}$ in the definition of $u_{b r}$ by Madsen et al. (1988) reduces the discrepancy to $13 \%$.

It is noted that $\overline{\mathcal{D}}_{f, M P G}$ and $\overline{\mathcal{D}}_{f}$ have identical spectral shapes.

\subsection{Comparison with Chen and Zhao (2012)}

Chen and Zhao (2012) presented two theoretical models for the energy dissipation within a canopy. The first of these is qualitatively similar to the present work, but they apply a linearisation like Madsen et al. (1988). The model was used by Jadhav et al. (2013) to derive frequency dependent drag coefficients on field data. It was found in the preceeding section that the linearisation results in a 1.13 times larger dissipation per vegetation layer with the present model. The ratio $1 / 1.13=0.88$ is close to the recommended correction factor of 0.85 to account for directional spreading (Section 5.1), so it appears that Chen and Zhao (2012) unintentionally accounted for directional spreading in their formulation. The frequency distribution of the dissipation by Chen and Zhao (2012) also accounts for the experimentally identified cut-off frequency.

The second approach is based on a joint distribution of sets of individual wave heights and individual wave periods, where the dissipation for each set is determined according to an expression similar to the Dalrymple et al. (1984) dissipation, $\overline{\mathcal{D}}_{D K H}$. The consequence is that the peak in the dissipation moves to frequencies noticeably higher than the peak frequency; an observation that cannot be recreated with the present formulation, which is based directly on the wave-induced velocity signal. It is the impression of the authors that the use of an intermediate step with joint distributions of wave periods and heights is not justified.

\subsection{Recommendations}

It is noted that the existing models of Mendez and Losada (2004) and Suzuki et al. (2012) model the dissipation due to 
vegetation well in conditions with $k_{p} h<1$ and emergent vegetation. These conditions are commonly present during storms. The present model more accurately captures the underlying physics for dissipation from submerged vegetation in milder wave conditions $(k h>1)$. These milder wave conditions are particularly important for modelling hydrodynamic conditions conducive for mud flat accretion.

The current work presented a new dissipation model, which eliminates the need to define a characteristic velocity profile (characteristic set of $\omega$ and $k$ ). It is recommended to apply this model, and existing experimental data should be re-fit to this model in order to define a consistent set of drag coefficients.

The problem with Suzuki et al. (2012) and - in particular Mendez and Losada (2004) is that the derived drag coefficients become functions of the degree of submergence and spectral width. Therefore, it is hard to judge the range of applicability of the drag coefficients. Optimally, the velocity reduction factor, $\alpha_{u}$, should also be incorporated, but many experimental campaigns have only measured the surface elevation, so it is better to assume $\alpha_{u}=1$, which is also done in The SWAN Team (2013).

The present model can be used both in bulk and as a frequency distributed model and it is recommended to apply it as a substitute for any dissipation models that are based on a characteristic velocity profile.

\section{Conclusion}

The new model for wave energy dissipation is presented in closed form for a given vertical level and instructions for vertical integration of the model over the height of the canopy are given. The present model resolves the issues with a characteristic velocity profile applied in existing and popular dissipation models. The frequency cut-off, commonly found in bottom friction energy dissipation models, where shorter waves are locally in deep water and do not experience energy dissipation from submerged vegetation is implicitly accounted for in this model. This new model offers a more realistic frequency distribution of the dissipation.

The approach to evaluate the drag coefficient was found highly important and it is concluded that the bulk drag coefficient based on one approach cannot be universally applied to any other spectral dissipation model. It was found to be possible to apply drag coefficients derived with Mendez and Losada (2004) in the spectral implementation in SWAN (Suzuki et al., 2012) for submerged vegetation cases with $k_{p} h<0.7, \gamma \geq 1.0$ and $h_{v} / h \geq 0.25$. Outside this range of validity, the dissipation is likely not captured correctly and the effect of the characteristic velocity profile will have a marked influence on the frequency distribution of the dissipation. The effect of the dissipation model choice is smallest for the emergent vegetation.

The new model was validated against experimental data. An experimental campaign was conducted at the University of Aberdeen, where submerged vegetation with different flexibilities and lengths were studied. The dissipation distribution between the present model and the Suzuki et al. (2012) model were compared to each other and the normalized spectral gradient was studied to understand the frequency dependent spectral energy dissipation. The shapes of the dissipation distribution differed significantly for shortest vegetation $\left(h_{v} / h=0.38\right)$ with the present model having a larger dissipation at frequencies less than the spectral peak and a steeper tail after the peak which always decreased to zero before the frequency cut-off. Analysis of the normalized spectral gradient corroborated the results of the present model. The ratio of total dissipation at each wave gauge using SWAN's Suzuki model compared to the present model was in the range $0.74 \leq \overline{\mathcal{D}}_{S W A N} / \overline{\mathcal{D}}_{v, \text { tot }} \leq 0.87$ for all tests with $h_{v} / h=0.38$.

The improvements of the present model are particularly important for modelling milder wave climates with submerged vegetation, as highlighted by the application to the experimental data. This model can be used as a bulk or frequency distributed model, and is recommended for substitution of dissipation models based on characteristic velocity profiles.

\section{Acknowledgements}

Robert C. Houseago (University of Hull) conducted the data collection with assistance from University of Aberdeen students Ross Horgan and Rory Summers. The artificial vegetation flexibilities and densities were designed by Robert C. Houseago as part of his University of Hull funded $\mathrm{PhD}$ study on the effect of flexibility on in-canopy hydrodynamics. The University of Aberdeen wave flume experiments were supported by the European Community's Horizon 2020 Programme through the grant to the budget of the Integrated Infrastructure Initiative HYDRALAB+, Contract no. 654110. The support of Mary Anderson Bryant and Jane Smith for providing their insight is graciously acknowledged. The support of the Coastal Structures and Wave Department of Deltares is acknowledged. This work was funded by the U.S. Department of Defense and Deltares through the Engineer and Scientist Exchange Program (ESEP) and the U.S. Army Corps of Engineers through the Engineering With Nature ${ }_{\circledast}\left(\mathrm{EWN}_{\circledast}\right)$ initiative.

\section{Appendix A. Convergence of vertical integration}

The frequency distributed dissipation at a given level was derived in closed form, however, numerical integration is required over the height of the vegetation. It is suggested to apply Simpson's integration rule to this and the number of required integration points is eluded to in this appendix.

The required number of (odd) integration points to achieve an error $\epsilon_{M}<0.001$ is investigated, where

$$
\epsilon_{M}=\frac{\left[\bar{d}_{v}\right]_{z, M}}{\left[\bar{d}_{v}\right]_{z, 1501}}
$$

$[\cdot]_{z, M}$ means numerical integration over $z$ with Simpson's rule with $M$ points and $M=1501$ is taken as the target. The result is depicted in Figure A.14A and it is seen that a large part 

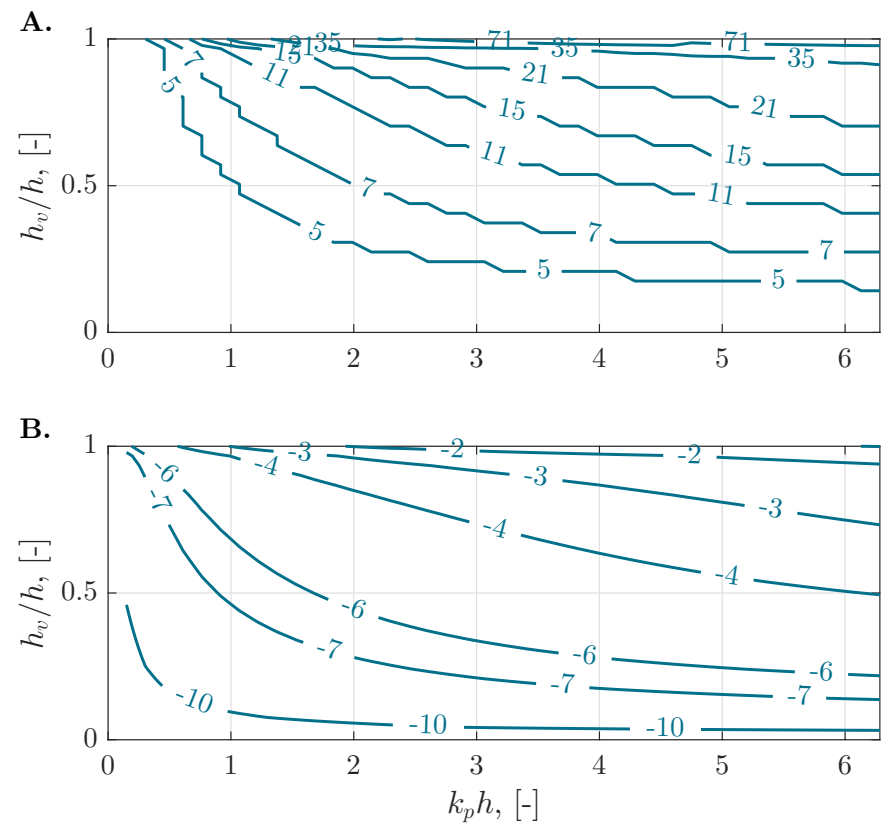

Figure A.14: Number of integration points with Simpson's integration rule. A: Required points for $\epsilon_{M}<0.001$. B: $\log _{10} \epsilon_{M=21}$.

of the domain $k_{p} h$ and $h_{v} / h$ is accurately described with $15-21$ integration points.

However, a larger number of integration points are required for large values of $k_{p} h$ and $h_{v} / h$. This is due to the fact that the frequencies with large wave numbers and large gradients over the height gets increasing relevance for the bulk dissipation. However, it is not feasible to analyse the number of required points in a large spectral model per computational point, so the committed error for $M=21$ is shown in Figure A.14B. It shows that $\epsilon_{M}$ only exceeds 0.01 for near-emergent vegetation and $\pi<k_{p} h$. This appears acceptable, but the correct number of integration points will always rely on an engineering judgement.

An alternative is to apply Simpson's rule on an nonequidistant vertical axis and base the point distribution on the most influencial wave number. A thorough analysis of an optimised integration scheme is deemed outside the scope of this work.

\section{References}

Anderson, M. E., Smith, J. M., 2014. Wave attenuation by flexible, idealized salt marsh vegetation. Coastal Engineering 83, 82-92.

Anderson, M. E., Smith, J. M., 2015. Implementation of wave dissipation by vegetation in stwave. Tech. rep., ERDC/CHL CHETN-I-85. Vicksburg, MS: U.S. Army Engineer Research and Development Center.

Cao, H., Feng, W., Hu, Z., Suzuki, T., Stive, M. J. F., 2015. Numerical modeling of vegetation-induced dissipation using an extended mild-slope equation. Ocean Engineering 110, 258-269.

Cavallaro, L., Re, C. L., Paratore, G., Viviano, A., Foti, E., 2010. Response of Posidonia oceanic to wave motion in shallow-waters: Preliminary experimental results. In: Proceedings of the 32nd International Conference on Coastal Engineering. Coastal Engineering Research Council, pp. 1-10.

Chen, Q., Zhao, H., 2012. Theoretical models for wave energy dissipation caused by vegetation. Journal of Engineering Mechanics 138 (2), 221-229.
Dalrymple, R. A., Kirby, J. T., Hwang, P. A., 1984. Wave diffraction due to areas of energy dissipation. Journal of Waterway, Port, Coastal and Ocean Engineering 110 (1), 67-79.

Hu, Z., Suzuki, T., Zitman, T., Uittewaal, W., Stive, M., 2014. Laboratory study on wave dissipation by vegetation in combined current-wave flow. Coastal Engineering 88, 131-142.

Jacobsen, N. G., 2016. Wave-averaged properties in a submerged canopy: Energy density, energy flux, radiation stresses and Stokes drift. Coastal Engineering 117, 57-69.

Jadhav, R. S., Chen, Q., Smith, J. M., 2013. Spectral distribution of wave energy dissipation by salt marsh vegetation. Coastal Engineering 77, 99-107.

Jadhav, R. S., Chen, Q., Smith, J. M., 2013. Spectral distribution of wave energy dissipation by salt marsh vegetation. Coastal Engineering 77, 99-107.

Losada, I. J., Maza, M., Lara, J. L., 2016. A new formulation for vegetationinduced damping under combined waves and currents. Coastal Engineering $107,1-13$

Lovas, S. M., 2000. Hydro-physical conditions in kelp forests and the effect on wave dumping and dune erosion: A case study on Laminaria hyperborea. Ph.D. thesis, University of Trondheim, The Norwegian Institute of Technology.

Lowe, R. J., Falter, J. L., Koseff, J. R., Monismith, S. G., Atkinson, M. J., 2007. Spectral wave flow attenuations within submerged canopies: Implications for wave energy dissipation. Journal of Geophysical Research, C05018.

Lowe, R. J., Koseff, J. R., Monismith, S. G., 2005. Oscillatory flow through submerged canopies: 1. velocity structure. Journal of Geophysical Research, C10016.

Ma, G., Kirby, J. T., Su, S.-F., Figlus, J., Shi, F., 2013. Numerical study of turbulence and wave damping induced by vegetation canopies. Coastal Engineering 80, 68-78.

Madsen, O. S., Poon, Y.-K., Graber, H. C., 1988. Spectral wave attenuation by bottom friction: Theory. Proceeding to International Conference on Coastal Engineering, 492-504.

Maza, M., Lara, J. L., Losada, I. J., Ondiviela, B., Trinogga, J., Bouma, T. J., 2015. Large-scale 3-D experiments of wave and current interaction with real vegetation. Part 2: Experimental analysis. Coastal Engineering 106, 73-86.

Mendez, F. J., Losada, I. J., 2004. An empirical model to estimate the propagation of random breaking and nonbreaking waves over vegetation fields. Coastal Engineering 51, 103-118.

Méndez, F. J., Losada, I. J., Losada, M. A., 1999. Hydrodynamics induced by wind waves in a vegetation field. Journal of Geophysical Research 104 (C8), 18,383-18,396.

Möller, I., Kudella, M., Rupprecht, F., Spencer, T., Paul, M., Van Wesenbeeck, B. K., Wolters, G., K., J., Bouma, T. J., Miranda-Lange, M., Schimmels, S., 2014. Wave attenuation over coastal salt marches under storm surge conditions. Nature Geoscience 7, 727-731.

Ozeren, Y., Wren, D. G., Wu, W., 2014. Experimental investigation of wave attenuation through model and live vegetation. Journal of Waterway, Port, Coastal, and Ocean Engineering 140 (5), 04014019.

Sánchez-González, J. F., Sánchez-Rojas, V., Memos, C. D., 2011. Wave attenuation due to Posidonia oceanica meadows. Journal of Hydraulic Research 49 (4), 503-514.

Smit, M. W. J., Reniers, A. J. H. M., Stive, M. J. F., 2012. Role of morphological variability in the evolution of nearshore sandbars. Coastal Engineering 69, 19-28.

Suzuki, T., Zijlema, M., Burger, B., Meijer, M. C., Narayan, S., 2012. Wave dissipation by vegetation with layer schematization in SWAN. Coastal Engineering 59, 64-71.

The SWAN Team, 2013. SWAN - Scientific and Technical Documentation. Delft University of Technology, Faculty of Civil Engineering and Geosciences, swan cycle iii, version $40.91 \mathrm{abc}$ Edition.

Van Rooijen, A. A., Lowe, R. J., Ghisalberti, M., Hansen, J., McCall, R. T., Van Dongeren, A. R., 2016. Physical and numerical modelling of wave transformation through a coastal canopy. 20th Australian Fluid Mechanics Conference, 1-4.

Van Rooijen, A. A., McCall, R. T., Van Thiel de Vries, J. S. M., Van Dongeren, A. R., Reniers, A. J. H. M., Roelvink, J. A., 2016. Wave attenuation and wave setup reduction due to aquatic nearshore vegetation. Journal of Geophysical Research - Oceans (DOI:10.1002/2015JC011392).

Wu, W.-C., Cox, D. T., 2015. Effects of wave steepness and relative water depth on wave attenuation by emergent vegetation. Estuarine, Coastal and Shelf Science $164,443-450$. 
Wu, W.-C., Ma, G., Cox, D. T., 2016. Modeling wave attenuation induced by the vertical density variations of vegetation. Coastal Engineering 112, 1727. 